\title{
A Survey on Particle Swarm Optimization for Use in Distributed Generation Placement and Sizing
}

\author{
Syed Muhammad Arif ${ }^{1, a}$, Akhtar Hussain ${ }^{2}$ and Dong Ryeol Shin ${ }^{1}$ \\ ${ }^{1}$ College of Information and Communication Engineering Sungkyunkwan University, Suwon, South Korea \\ ${ }^{2}$ Department of Electrical Engineering, Incheon National University, Incheon, South Korea
}

\begin{abstract}
This paper surveys the research and development of Particle Swarm Optimization (PSO) algorithm for use in selecting a suitable position for Distributed Generation (DG) units within a distribution network. Our discussion first covers the algorithm development of PSO and its use in neural networks. After establishing the foundations of PSO, we then explore its use in sizing and sitting of DG units in distribution network. Combining PSO with other optimization techniques for attaining better results is also discussed in this paper.
\end{abstract}

\section{Introduction}

In the conventional power system, the generating units are kept far away from the consumers. While it has certain advantages, the biggest disadvantage is the occurrence of heaving line losses when power is transported to the consumers. There can be many ways of reducing power loss in the distribution network. For example, the use of superconductors in transmission lines can reduce line losses. However, the superconductor technology is still in its early days and still quite expensive. More recently, the concept of Distributed Generation (DG) has come up which is environmentally friendly and economical. The sizing and placement of DG units can significantly affect the system losses. This has initiated considerable interest in finding an optimum position of the DG unit. To find a suitable place for a DG unit in Smart Grids, the authors in [1] use the Multi Criteria Decision Analysis. The optimization variables include Voltage Stability Index (VSI), Frequency Deviation in Substation (FDSS) and Frequency Deviation in DG (FDDG). The same authors in [2] have used circuit analysis to detemine the voltage stability index (VSI) for distribution networks connected in loop configuration.In this paper, we are more concerned with exploring PSO for optimizing the sizing and siting of DG units. Such an optimization problem canbe solved by different optimization algorithm [3], for instance Tuba Search (TS). However, is a time consuming algorithm and has a tendency to get trapped into local optimal value. Search Annealing (SA) is another possible algorithm which also consumes considerable time to get the optimal solution. As compared to TS and SA, Genetic Algorithm (GA) has been widely used for finding the global (or near global) optimal solution of the problem. PSO has been proven to be better than GA in terms of computational time and convergence speed [3]. Hence, this paper surveys the use of PSO in placing DG units.

\section{Original PSO algorithm}

The original PSO algorithm, which is a non-linear optimization algorithm, was discovered by James Kennedy and Russell Eberhart in 1995 [4], [5] while observing the social behaviour of animals and birds. The authors proposed the velocity and position equations for each particle travelling in a group. The prevailing velocity can be calculated by using the previous velocity and the distance between $P_{\text {best }}$ and $G_{\text {best }}$ as expressed by following formula [4], [5]. All particles adjust their position according to the personal flying experience called $\mathrm{P}$ best (personal best) as well as the flying experience of the other particles in the group called $G$ best (Global best).

$$
v_{i}^{k+1}=v_{i}^{k}+c_{1} r_{1}\left(\text { Pbest }_{i}^{k}-x_{i}^{k}\right)+c_{2} r_{2}\left(\text { gbest }^{k}-x_{i}^{k}\right)
$$

The current position can be calculated by adding the current velocity in previous position.

$$
x_{i}^{k+1}=x_{i}^{k}+v_{i}^{k+1}
$$

$V_{i}^{k}$ : Velocity of particle i at iteration $\mathrm{k}$

$C 1$ : Cognitive factor; $C 2$ : Social factor

$\mathrm{r} 1, \mathrm{r} 2$ : Uniformly distributed random number between $0-1$

$x_{i}^{k}$ : Position of particle $\mathrm{i}$ at iteration $\mathrm{k}$

Pbest $_{i}{ }^{k}$ : Personal best of agent $\mathrm{i}$ at iteration $\mathrm{k}$

Gbest ${ }^{k}$ : Best among all in the group

Kennedy and Eberhart [4], [5] have proposed the value of $\mathrm{c} 1=\mathrm{c} 2=2$. If $\mathrm{c} 1$ is much greater than $\mathrm{c} 2$, each

\footnotetext{
${ }^{\mathrm{a}}$ Corresponding author.
} 
particle is remains attracted to its own personal best causing excessive wandering. Consequently, particles can not find the optimal solution and remain trapped in the local value. On the other hand, if $\mathrm{c} 2$ is much greater than $\mathrm{c} 1$, the particles are strongly attracted towards the global best position. This is also undesirable because the particles may move away from the optimal value.

All the particles in a search space should obey these three rules [6].

1. Avoid collision with the neighbours

2. Match their velocity with that of the neighbours

3. Stay in close vicinity with the neighbours

\subsection{Modified PSO algorithm}

The author in [7] modified the velocity equation by introducing the construction factor ' $\mathrm{K}$ ' to insure the convergence of PSO. The modified expression is as follows:

$$
v_{i}^{k+1}=K\left[v_{i}^{k}+c_{1} r_{1}\left(\operatorname{Pbest}_{i}^{k}-x_{i}^{k}\right)+c_{2} \boldsymbol{r}_{2}\left(\text { gbest }^{k}-x_{i}^{k}\right)\right]
$$

where $K=\frac{2}{\left|2-\varphi-\sqrt{\varphi^{2}-4 \varphi}\right|}$ where $\varphi=C 1+C 2, \varphi>4$

The authors in [8] introduced a new parameter called the inertia weight in the standard PSO form:

$$
\begin{gathered}
v_{i}^{k+1}=w v_{i}^{k}+c_{1} r_{1}\left(\text { Pbest }_{i}^{k}-x_{i}^{k}\right)+c_{2} r_{2}\left(\text { gbest }^{k}-x_{i}^{k}\right) \\
v_{i}^{k+1}=w v_{i}^{k}+c_{1} r_{1}\left(\text { Pbest }_{i}{ }^{k}-x_{i}^{k}\right)+c_{2} \boldsymbol{r}_{2}\left(\text { gbest }^{k}-x_{i}^{k}\right)
\end{gathered}
$$

Refer to right side of equation (3a), which consists of three parts: the first part shows the previous velocity, the second and third parts are called the cognitive and social part. Without the second and third parts the particles keep on flying with the current velocity. Cognitive part increase the local search ability and social part increase the global search ability. The inertia weight $\mathrm{w}$ in Equation (3a) balances the local and global search abilities. The main advantage of introducing $\mathrm{w}$ is that it eliminates the requirement of careful setting of velocity. Also, increasing the inertia weight helps the search for a global solution and vice versa.

The authors in [9] have used $\mathrm{w}=0.7$ with the hit and trial method to balance the global and local search abilities.

The authors in [10] have used variable weight factor for the optimal design of small renewable energy systems. In a search space particles are flying to find the optimal solution. The process of reaching the particles towards a common point is called convergence. The proposed method keeps on increasing the weight until convergence is achieved. This modified PSO technique shows faster convergence speed and needs less computational time as compared to the standard PSO technique. Mathmatically, the variable inertia weight is represented as follows:

$$
w=\frac{\left(\mathcal{W}_{\max }-\mathcal{W}_{\min }\right)}{\# \text { iteration }} * \text { current iteration no. }
$$

The PSO flow chart shown in Figure 1, detail discussion of flow chart is beyond the scope of this paper. Details are given in [31]

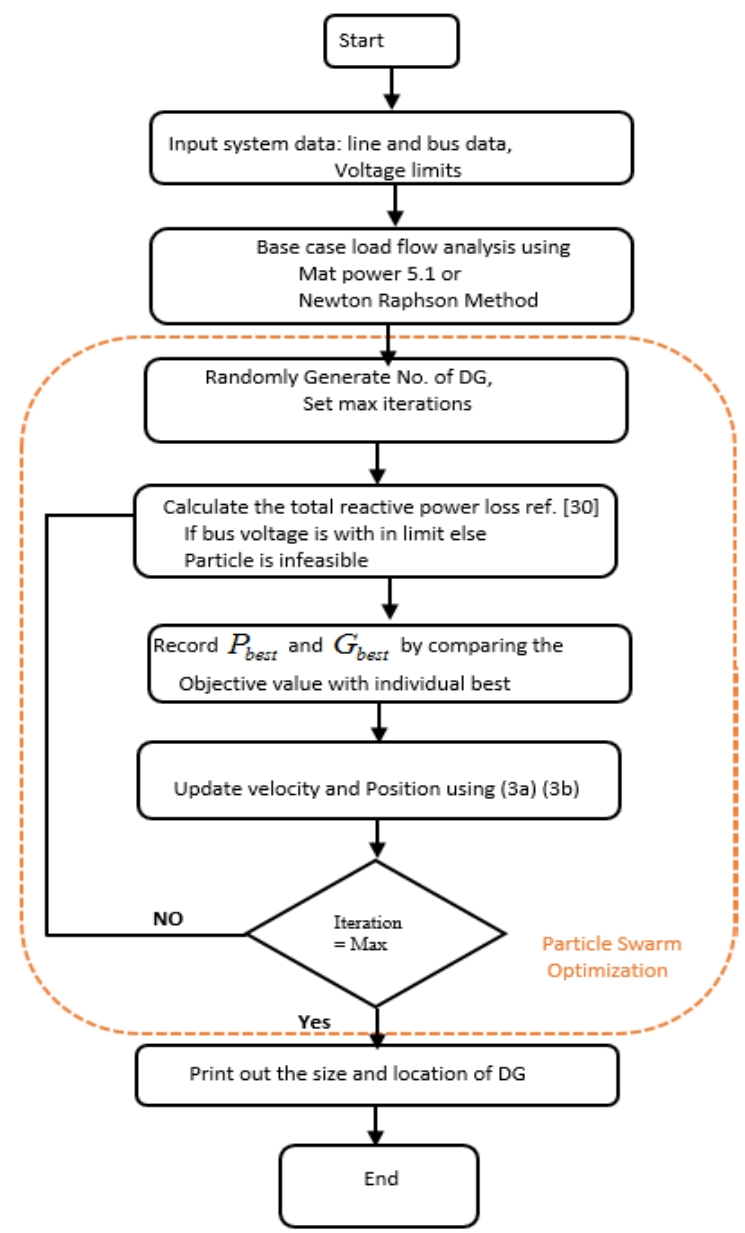

Figure 1. PSO flow chart.

\section{Training a neural network using PSO}

Data processing, classification and pattern recognition are widely used applications of Artificial Neural Network (ANN). ANN is a learning method motivated by the learning ability of human brain's neural systems which comprises of three layers structures: input, output and hidden layers. There are several learning algorithms, such as Back Propagation (BP) [11], Gradient Descendant [12], etc. One of the main disadvantages of these techniques is the slow convergence and tendency to get trapped in the local minima [13]. Since PSO is a population based search algorithm it can produce better results. One of the biggest issue with PSO is due to the strong competition between the flying particles in a search space the PSO get trap into local minima. To avoid getting trapped into the local minima and achieving fast convergence, the authors in [14] proposed Gaussian PSO with fuzzy reasoning based structural learning. Instead of uniformly distributed random variable, Gaussian random variable is proposed to escape from the local minima and the structural learning with fuzzy reasoning is proposed to improve the computational time by modifying the network structure. The authors in [15] propose optimization of type-2 fuzzy weights in back propagation learning for NN using PSO 
and GA. The type-2 fuzzy logic enhance the uncertainty in the learning while PSO and GA increases the convergence speed. It has been emphasized in [16], [17] that the weight affects the performance of the learning process. Another inspiring application of PSO in Neural Network is prostate cancer prediction system. The authors in [18] have proposed two-stage fuzzy neural network for prognosis system which provide more accurate prediction (due to fuzzy system) of prostate cancer results and translate it into information for doctor. [19] Shows the experimental study for pixel classification in satellite imaginary, the author did 1050 numbers of experiments and proposed the solution of two critical issues i.e. determination of hidden layers neurons and most discriminative spectral band. The multi-objective (MPSO) improve the classification accuracy and computation time.

\section{Using PSO for distributed generation}

Recent research shows that installing a DG unit in a distribution network leads to various technical, economical and enviromental benefits. However, in order to reap these benefits, it is very important to find an optimal location and size of a DG unit.There are a number of constraints that need to be considered before placing a DG unit [20], [37].

Equality constrains: Power flow equations represent the equality constrains (total power injected at any bus should be equal to total generation minus demand). For each bus the power flow equation should be satisfied, which can be handle by load flow such as Newton Raphson.

Inequality constrains: The thermal constrains and equiptment rating are called inequality constrains. These constrains can be handled by proper sizing the DG unit which can be done by using PSO. Inequality constrains are as follow [32]

1) Each segment in the distribution network has a thermal limits which should not exceed than its maximum rating.

2) The output of DG should not exceed then the power supplied by the substation.

3) The total number of DG connected in distribution network should be bounded by a certain number. By definition, only one DG unit can place at a given bus.

Boundary conditions: While placing the DG at a given bus the voltage magnitude and angle should be kept at a certain acceptable level.

\subsection{Optimal sizing}

The authors in [21] have used PSO for optimal sizing of the stand-alone hybrid power system. This system includes wind unit, electrolysers, a reformer, anaerobic reactor, fuel cell and some hydro tanks. The objective is to minimize the total cost of the system and the components have optimal sizes, such that the power demand by the system meets. It has been argued in [22][24] that PSO provides excellent convergence and feasible optimal solution for sizing the micro-grid system consisting of wind/PV/battery banks/ fuel cells and reformers. The comparative analysis in [25] focuses on the size of the off-grid Renewable Hybrid Energy System (micro hydro plant, fuel cell, wind turbine and solar panel etc.) using GA and PSO. The analysis shows that PSO is much better then GA in terms of CPU utilization and the number of iterations to find the optimal value. The authors in [26] proposed improved PSO which utilize the benefits of both the GA and PSO that can enhance global searching capability and convergence of particles. To improve the convergence rate the convergence factor $\mathrm{K}$ is introduce and the global search ability is enhance by introducing the idea of mutation of genetic algorithm this can escape the local trapping. In [27], the authors have proposed the structure of a micro grid consisting of Wind PV hybrid system with battery, Hydro tank and fuel cell. The author used PSO algorithm to achieve the best sizing of the DG unit for the proposed micro grid. The authors in [28] have proposed an approach for sizing the hybrid systems (Wind/PV/Diesel Generator/Fuel Cell/Battery and Hydro Tank). The $\mathcal{E}$-constrain method [29] is used to minimize the overall system cost and $\mathrm{CO}_{2}$ emission produced by the diesel generators. The author in [30] proposed an approach for sitting and sizing of four types of DG units to enhance the loadability of primary distribution systems. The results show that there is a strong correlation between reactive power loss and loadability of the system

\subsection{Optimal siting}

The author in [31] have proposed multi-objective based approach to find the best location and size of different DG units with different load model having non-unity power factor. A simple PSO algorithm is used to obtain the best solution for the multi objective problem. The authors in [32] have proposed improved PSO algorithm for the placement and sizing of multiple DG units. The problem is divided into two sub problems: DG unit's optimal location (discrete optimization) and its optimal size (continuous optimization). In the standard PSO, a factor is multiplied with inequality constrains and added into the objective function called penalty factor. The proper selection of penalty factor is very difficult which differ form problem to problem. The author eliminates the needs of proper selection of penalty factor by using a unique PSO feature. If the particle flies away from the search space then restore its violated position to $P_{b e s t}$ this keeps the infeasible particles alive as a candidate. Author in [33] use the application of PSO for DG sizing and siting in the radial distribution network. The objective is to improve the voltage profile, and active power compensation by reducing the real power loss. By using the exact loss formula, the authors calculate the size of DG unit at every bus. The location of the DG unit is calculated by using the loss sensitivity factor. The bus having lowest power loss and lowest sensitivity factor will be the best location for DG placement. The authors in [34] use PSO for optimal placement of different types of DG units which can supply both real and reactive power in the distribution networks. The proposed PSO 
approach is tested on IEEE-69 bus distribution system and the result shows that as compared to other heuristic approaches, the proposed algorithm gives smaller DG size. The authors in [35] have used the dynamic sensitivity analysis method for the placement of capacitors which will reduce the search space problem. The results show that for more than one location, dynamic sensitivity is good because it helps in deciding other positions considering the previous locations and the value of capacitor. The comparison results show that the proposed PSO approach provides better global solution with greater saving. The authors in [36] have used PSO for optimal allocation of DG units for multi-phase unbalanced distribution network. The results show that optimal size and allocation of DG unit can reduce the total power loss and improve the voltage profile in the distribution network. The proposed method can place DG unit more effectively and in less computation time. The power loss reduction using PSO is better than power loss reduction using RPF (repeated power flow).

\subsection{Hybrid PSO}

Researchers are focusing on the hybridization of PSO with other techniques for getting better results. The authors in [37] have proposed hybrid PSO/GA algorithm to minimize the network power loss, better voltage regulation, and improve voltage stability index. The result shows that hybrid PSO/GA has better power minimization as compared to PSO and GA while GA has better voltage profile improvement as compared to PSO and Hybrid PSO/GA. The authors concluded that hybrid method offer a higher ability of finding the optimal solution. The authors in [38] have proposed hybrid PSO with analytical method (AM) to reduce the power loss and to improve the voltage profile. The sizing is done by $\mathrm{AM}$ and the placement is done by PSO. The result shows that loss reduction becomes more effective by increasing the number of DG units in the network. It also increases the voltage profile. In [39] the authors have proposed hybrid PSO which has a problem of being trapped into local optima and take a lot of time for getting the optimal solution. To balance the local and global searching ability, the same authors [40] have used fuzzy adaptive inference for tuning the inertia weight of PSO. The result shows that fuzzy Adaptive Particle Swarm Optimization (FAPSO) gives the optimal solution after 69 iterations while other PSO method need 190 cycles. The authors in [41] proposed a novel hybrid Rank Evolutionary PSO to improve the computation time (fastest) of multiple DG. The results shows that Rank Evolutionary PSO has stable performance and fast result in moving the particle to global optimal solution, less number of iteration, lowest standard deviation and fastest computing time as compared to other PSOs.

\section{Conclusion}

This paper presents a summary of the research and development in Particle Swarm optimization algorithm especially for DG placement and sizing. It highlights the standard and modified algorithm and constraints needed to be handled when the DG unit is placed in the distribution network. Several applications of PSO in training a neural network, distribution network for DG sizing and siting and hybrid PSO are also discussed in the literature.

\section{Acknowledgement}

The author would like to express out appreciation to Prof. Faraz Hasan, Massey University, New Zealand for sharing their pearls of wisdom during the course of this research. This research was supported by the MISP (Ministry of Science, ICT \& Future Planning), Korea, under the National Program for Excellence in SW) (R2215-16-1005) Supervised by the IITP (Institute for Information \& communications Technology Promotion).

\section{References}

1. Kazmi, SAA., Hasan, SF., \& Shin, DR., "Multi Criteria Decision Analysis for Optimum DG Placement in Smart Grids", IEEE Innovative Smart Grids Technologies Conference (Asia) (2015)

2. Kazmi, SA., Hasan, SF., \& Shin, DR.,"Circuit Analysis Approach for Calculating Voltage Stability Index", IEEE International Conference on Power Electronics and Drive Systems (2015)

3. A.M. El-Zonkoly, "Optimal placement of multidistributed generation units including different load models using particle swarm optimisation" IET Gener.Transm. Distrib., 5, 7, pp. 760-771, (2011)

4. J. Kennedy and R. Eberhart, "Particle swarm optimization," in Proc. IEEE Int. Conf. Neural Netw., 4, pp. 1942-1948 (1995)

5. R. Eberhart and J. Kennedy, "A new optimizer using particle swarm theory," in Proc. 6th Int. Symp. Micro Machine Human Science, pp. 39-43 (1995)

6. Reynolds, C. W. Flocks, Herds, and Schools "A Distributed Behavioral Model, in Computer Graphics", 21(4) (SIGGRAPH) pages 25-34 (1987)

7. R. C. Eberhart and Y. Shi, "Comparing Inertia Weights and Constriction Factors in Particle Swarm Optimization" IEEE (2000)

8. Shi, Y. and Eberhart, R.C., "A modified particle swarm optimizer", Proceedings IEEE International Conference on Evolutionary Computation, 69-73. Piscataway, NJ: IEEE Press. (May 1998)

9. S.M. Hakimi and S.M. Moghaddas-Tafreshi, "Optimal sizing of a stand-alone hybrid power system via particle swarm Optimization for Kahnouj area in south-east of Iran", Renewable Energy, 34 1855-1862 (2009)

10. Ahmed Hassan, Magdi Saadawi, Mahmoud Kandil, Mohammed Saeed, "Modified particle swarm optimization technique for optimal design of small renewable energy system supplying a specific load at Mansoura University", IET Renew. Power Genre., 9, 5, pp. 474-483 (2015) 
11. Marco Gori, Alberto Tesi, "On the problem of local minima in back-propagation algorithm", Neural Netw., 5(4) 465-471 (1992)

12. M. F. Moller, "A Scaled Conjugate Gradient Algorithm for Fast Supervised Learning", PB-339 Reprint, Computer science Department, University of Aaur- hus, Denmark, (1990)

13. C.H.Chen, H.Lai, "Anempirical study of the Gradient descentand the conjugate gradient back propagation neural networks", in: Proceedings OCEANS' 92 Mastering the Oceans through Technology, 1, 1991, pp. 132-135 (1992)

14. Haydee Melo, Junzo Watada, "Gaussian-PSO with fuzzy reasoning based on structural learning for traininga Neura 1Network", Neurocomputing, 172405-412 (2016)

15. Fernando Gaxiola, Patricia Melina, Fevrier Valdez, Juan R. Castro, Oscar Castillo, "Optimization of type-2 fuzzy weights in backpropagation learning forneural networks using GAs and PSO", Applied Soft Computing, 38-860-871 (2016)

16. Mohammad Ali Ahmadi, "Connectionist approach estimates gas-oil relative permeability in petroleum reservoirs: Application to reservoir simulation", Fuel, 140-429-439 (2015)

17. Mohammad-Ali Ahmadi, Mohammad Reza Ahmadi, Seyed Moein Hosseini, Mohammad Ebadi, "Connectionist model predicts the porosity and permeability of petroleum reservoirs by means of petro-physicallogs: Application of artificial intelligence", Journal of Petroleum Science and Engineering 123-183-200 (2014)

18. Ren-Jieh Kuoa, Man-Hsin Huangb, Wei-Che Chenga, Chih-Chieh Linc,d, Yung-Hung Wue, "Application of a two-stage fuzzy neural network to a prostate cancer prognosis system", Artificial Intelligence in Medicine 63 -119-133 (2015)

19. Rajesh K. Agrawal, Narendra G. Bawane, "Multiobjective PSO based adaption of neural network topology for pixel classification in satellite imagery", Applied Soft Computing 28217-225 (2015)

20. Florina Rotaru, Gianfranco Chicco, Gheorghe Grigoras ,Gheorghe Cartina,"Two-stage distributed generation optimal sizing with clustering-based node selection", Electrical Power and Energy Systems 40 120-129 (2012)

21. H. Hassanzadehfard, S.M. Moghaddas-Tafreshi, S.M. Hakimi, "Optimal Sizing of an Islanded Micro-Grid for an Area in North-West Iran Using Particle Swarm Optimization Based on Reliability Concept", World Renewable Energy Congress -Sweden (2011)

22. S.M. Moghaddas-Tafreshi and S.M.Hakimi, "Optimal Sizing of a stand-alone Hybrid Power System via Particle Swarm Optimization (PSO)", International Power Engineering Conference -IPEC (2007)

23. H. Valizadeh Haghi, S. M. Hakimi, and S. M. Moghaddas Tafreshi ,"Optimal Sizing of a Hybrid Power System Considering Wind Power Uncertainty Using PSO-Embedded Stochastic Simulation", PMAPS (2010)
24. M. Bashir and J. Sadeh, "Size Optimization of New Hybrid Stand-alone Renewable Energy System Considering a Reliability Index", IEEE (2012)

25. Bhimsen Tudu, Sibsankar Majumder, Kamal K. Mandal, and Niladri Chakraborty,"Comparative Performance Study of Genetic Algorithm and Particle Swarm Optimization Applied on Off-grid Renewable Hybrid Energy System", Springer-Verlag Berlin Heidelberg (2011)

26. Y. S. Zhao, J. Zhan, Y. Zhang, D. P. Wang and B. G. Zou,"The optimal capacity configuration of an independent wind/Pv hybrid power supply system based on improved pso algorithm" (2009)

27. Arash Navaeefard, S.M.Moghaddas Tafreshi, Mostafa Barzegari, Amir Jalali Shahrood, "Optimal Sizing of Distributed Energy Resources in Microgrid Considering Wind Energy Uncertainty with Respect to Reliability", International Energy Conference IEEE (2010)

28. Masoud Sharafi, Tarek Y. ELMekkawy,"Multiobjective optimal design of hybrid renewable energy systems using PSO-simulation based approach", Renewable Energy 68-67-79 (2014)

29. Kaveh Khalili-Damghani, Amir-Reza Abtahi, Madjid Tavana, "A new multi-objective particle swarm optimization method for solving reliability redundancy allocation problems", Reliability Engineering and System Safety 111 -58-75 (2013)

30. Nguyen Cong Hien, Nadarajah Mithulananthan, Senior Member, IEEE, and R. C. Bansal, Senior Member, IEEE, "Location and Sizing of Distributed Generation Units for Loadabilty Enhancement in Primary Feeder", IEEE systems journal, 7, 4, (December 2013)

31. A. M. El-Zonkoly,"Optimal placement of multidistributed generation units including different load models using particle swarm optimization", Swarm and Evolutionary Computation 1 50-59 (2011)

32. M. R. AlRashidi , M. F. AlHajri, "Optimal planning of multiple distributed generation sources in distribution networks: A new approach", Energy Conversion and Management 52 3301-3308 (2011)

33. Satish Kansal, B.B.R. Sai, Barjeev Tyagi, Vishal Kumar, "Optimal placement of distributed generation in distribution networks", International Journal of Engineering, Science and Technology, 3, 3, pp. 4755 (2011)

34. Satish Kansal, Vishal Kumar, Barjeev Tyagi, "Optimal placement of different type of DG sources in distribution Networks", Electrical Power and Energy Systems 53-752-760 (2013)

35. S.P. Singh and A.R. Rao, "Optimal allocation of capacitors in distribution systems using particle swarm optimization", Electrical Power and Energy Systems 43-1267-1275 (2012)

36. Samir Dahal, Hossein Salehfar, "Impact of distributed generators in the power loss and voltage profile of three phase unbalanced distribution network", Electrical Power and Energy Systems 77256-262 (2016)

37. M.H. Moradi , M. Abedini, "A combination of genetic algorithm and particle swarm optimization 
for optimal DG location and sizing in distribution systems", Electrical Power and Energy Systems 3466-74 (2012)

38. Satish Kansal,Vishal Kumar, Barjeev Tyagi, "Hybrid approach for optimal placement of multiple DGs of multiple types in distribution networks", Electrical Power and Energy Systems 75-226-235 (2016)

39. Shuheng Chen, Weihao Hu, Chi Su, Xiaoxu Zhang, Zhe Chen, "Optimal reactive power and voltage control in distribution networks with distributed generators by fuzzy adaptive hybrid particle swarm optimisation method", IET Gener. Transm. Distrib., 9, 11, pp. 1096-1103 (2015)

40. Wen Shan Tan, Mohammad Yusri Hassan, Hasimah Abdul Rahman, Md Pauzi Abdullah, Faridah Hussin,"Multi-distributed generation planning using hybrid particle swarm optimization- gravitational search algorithm including voltage rise issue",IET Gener. Transm. Distrib., 7, 9, pp. 929-942 (2013)

41. J. J. Jamian, M. W. Mustafa, H. Mokhlis, "Optimal multiple distributed generation output through rank evolutionary particles warm optimization", Neurocomputing 152-190-198 (2015) 